\title{
Two years of INTEGRAL monitoring of the soft gamma-ray repeater SGR 1806-20: from quiescence to frenzy ${ }^{\star}$
}

\author{
D. Götz ${ }^{1}$, S. Mereghetti ${ }^{1}$, S. Molkov ${ }^{2,3}$, K. Hurley ${ }^{4}$, I. F. Mirabel ${ }^{5}$, R. Sunyaev ${ }^{2,3}$, G. Weidenspointner ${ }^{6}$, \\ S. Brandt ${ }^{7}$, M. Del Santo ${ }^{8}$, M. Feroci ${ }^{8}$, E. Göğ̈̈iş ${ }^{9}$, A. von Kienlin ${ }^{3}$, M. van der Klis ${ }^{10}$, C. Kouveliotou ${ }^{11,12}$, \\ N. Lund ${ }^{7}$, G. Pizzichini ${ }^{13}$, P. Ubertini ${ }^{8}$, C. Winkler ${ }^{14}$, and P. M. Woods ${ }^{15}$
}

1 INAF - Istituto di Astrofisica Spaziale e Fisica Cosmica, via Bassini 15, 20133 Milano, Italy e-mail: diego@mi.iasf.cnr.it

2 Space Research Institute, Russian Academy of Sciences, Profsoyuznaya 84/32, 117997 Moscow, Russia

3 Max Planck Institut für Astrophysik, Karl Schwarzschild Str. 1, 85740 Garching bei München, Germany

${ }^{4}$ University of California at Berkeley, Space Sciences Laboratory, Berkeley CA 94720-7450, USA

5 European Southern Observatory, Alonso de Cordova 3107, Santiago, Chile

${ }^{6}$ External ESA Fellow - Centre d'Étude Spatiale des Rayonnements (CESR), avenue du Colonel-Roche 9, 31028 Toulouse Cedex 4, France

7 Danish National Space Center (DNSC), Juliane Maries Vej 30, 2100 Copenhagen, Danemark

8 INAF - Istituto di Astrofisica Spaziale e Fisica Cosmica, via Fosso del Cavaliere 100, 00133 Roma, Italy

9 Sabanci University, Orhanli-Tuzla, Istanbul, Turkey

10 Astronomical Institute "Anton Pannekoek", University of Amsterdam and Center for High-Energy Astrophysics, Kruislaan 403, 1098 SJ Amsterdam, The Netherlands

11 National Space Science and Technology Center, 320 Sparkman Drive, Huntsville, AL 35805, USA

12 NASA Marshall Space Flight Center, XD 12, Huntsville, AL 35812, USA

13 INAF - Istituto di Astrofisica Spaziale e Fisica Cosmica, via Gobetti 101, 40129 Bologna, Italy

14 ESA-ESTEC, RSSD, Keplerlaan 1, 2201 AZ Nordwijk, The Netherlands

15 Department of Physics, University of Manchester, Sackville Street, PO Box 88, Manchester M60 1QD, UK

Received 17 June 2005 / Accepted 29 August 2005

\section{ABSTRACT}

SGR 1806-20 has been observed for more than 2 years with the INTEGRAL satellite. In this period the source went from a quiescent state into a very active one culminating in a giant flare on December 27, 2004. Here we report on the properties of all the short bursts detected with INTEGRAL before the giant flare. We derive their number-intensity distribution and confirm the hardness-intensity correlation for the bursts found by Götz et al. (2004a, A\&A, 417, L45). Our sample includes a very bright outburst that occurred on October 5, 2004, during which over one hundred bursts were emitted in 10 minutes, involving an energy release of $3 \times 10^{42} \mathrm{erg}$. We present a detailed analysis of it and discuss our results in the framework of the magnetar model.

Key words. gamma rays: bursts - gamma rays: observations - stars: pulsars: general - stars: pulsars: individual: SGR 1806-20

\section{Introduction}

SGR 1806-20 is currently one of the most active Soft Gamma-ray Repeaters. These sources (see Hurley (2000), Woods \& Thompson (2004) for recent reviews) were discovered by their recurrent emission of soft $(\leq 100 \mathrm{keV})$ gamma-ray bursts. They undergo sporadic, unpredictable periods of activity, which last days to months, often followed by long periods (up to years or decades) during which no bursts are detected.

^ Based on observations with INTEGRAL, an ESA project with instruments and science data centre funded by ESA member states (especially the PI countries: Denmark, France, Germany, Italy, Switzerland, Spain), Czech Republic and Poland, and with the participation of Russia and the USA.
These recurrent bursts have typical durations of $\sim 0.1 \mathrm{~s}$ and luminosities in the range $10^{39}-10^{42} \mathrm{erg} \mathrm{s}^{-1}$. Occasionally, SGRs also emit giant flares that last up to a few hundred seconds and exhibit remarkable pulsations that reveal their spin periods (e.g. Mazets et al. 1979; Hurley et al. 1999; Hurley et al. 2005).

The spin period of SGRs can also be measured in their persistent (quiescent) X-ray emission. Typical luminosities $(0.5-10 \mathrm{keV})$ of these heavily absorbed sources are of the order of a few $10^{35} \mathrm{erg} \mathrm{s}^{-1}$. Persistent emission from SGR 1806-20 has been detected recently also in the soft $\gamma$-ray range up to $\sim 150 \mathrm{keV}$ (Mereghetti et al. 2005a; Molkov et al. 2005).

The bursting activity and the persistent emission are generally explained in the framework of the "magnetar" 
Table 1. Observation summary of the bursts from SGR 1806-20 analysed here. (P: Public Data, CP: Core Program Observations, TOO: Target of Opportunity Observations, R: Open time Galactic Centre Data). The double horizontal line indicates the separation between the periods of different activity of the source (see text).

\begin{tabular}{ccccc}
\hline $\begin{array}{c}\text { INTEGRAL } \\
\text { orbit }\end{array}$ & $\begin{array}{c}\text { Obs. } \\
\text { type }\end{array}$ & $\begin{array}{c}\text { Number } \\
\text { of bursts }\end{array}$ & $\begin{array}{c}\text { Exposure } \\
\text { time [ks] }\end{array}$ & $\begin{array}{c}\text { Orbit start } \\
\text { time [UT] }\end{array}$ \\
\hline 56 & $\mathrm{P}$ & 1 & 90.1 & $2003-03-29 \mathrm{~T} 21: 37: 59$ \\
105 & $\mathrm{P}$ & 8 & 207.8 & $2003-08-23 \mathrm{~T} 10: 24: 56$ \\
108 & $\mathrm{P}$ & 2 & 215.5 & $2003-09-01 \mathrm{~T} 09: 41: 20$ \\
109 & $\mathrm{P}$ & 2 & 217.2 & $2003-09-04 \mathrm{~T} 09: 26: 46$ \\
114 & $\mathrm{P}$ & 1 & 208.7 & $2003-09-19 \mathrm{~T} 08: 27: 22$ \\
119 & $\mathrm{P}$ & 1 & 74.8 & $2003-10-04 \mathrm{~T} 07: 22: 32$ \\
120 & $\mathrm{P}$ & 4 & 201.2 & $2003-10-07 \mathrm{~T} 07: 11: 55$ \\
121 & $\mathrm{P}$ & 2 & 140.7 & $2003-10-10 \mathrm{~T} 07: 02: 43$ \\
122 & $\mathrm{P}$ & 22 & 201.3 & $2003-10-13 \mathrm{~T} 06: 53: 37$ \\
171 & $\mathrm{CP}$ & 1 & 197.9 & $2004-03-07 \mathrm{~T} 22: 21: 10$ \\
173 & $\mathrm{CP}$ & 2 & 175.5 & $2004-03-13 \mathrm{~T} 21: 54: 43$ \\
175 & $\mathrm{CP}$ & 2 & 72.1 & $2004-03-19 \mathrm{~T} 21: 30: 35$ \\
181 & $\mathrm{CP} / \mathrm{R}$ & 3 & 134.8 & $2004-04-06 \mathrm{~T} 20: 15: 13$ \\
\hline $56-181$ & & 51 & 2138 & \\
\hline \hline 225 & $\mathrm{TOO}$ & 41 & 169.9 & $2004-08-16 \mathrm{~T} 09: 51: 26$ \\
226 & $\mathrm{CP}$ & 5 & 32.1 & $2004-08-19 \mathrm{~T} 09: 37: 14$ \\
227 & $\mathrm{R}$ & 59 & 148.2 & $2004-08-22 \mathrm{~T} 09: 22: 35$ \\
229 & $\mathrm{CP}$ & 1 & 68.1 & $2004-08-28 \mathrm{~T} 08: 53: 12$ \\
230 & $\mathrm{R}$ & 2 & 208.5 & $2004-08-31 \mathrm{~T} 08: 41: 37$ \\
232 & $\mathrm{CP}$ & 1 & 52.1 & $2004-09-06 \mathrm{~T} 08: 17: 45$ \\
233 & $\mathrm{CP}$ & 2 & 36.2 & $2004-09-09 \mathrm{~T} 08: 04: 02$ \\
234 & $\mathrm{CP}$ & 1 & 14.8 & $2004-09-12 \mathrm{~T} 07: 50: 28$ \\
235 & $\mathrm{CP}$ & 2 & 77.9 & $2004-09-15 \mathrm{~T} 07: 37: 33$ \\
236 & $\mathrm{R}$ & 8 & 178.1 & $2004-09-18 \mathrm{~T} 07: 24: 13$ \\
237 & $\mathrm{R}$ & 2 & 173.2 & $2004-09-21 \mathrm{~T} 07: 09: 49$ \\
240 & $\mathrm{CP}$ & 8 & 208.7 & $2004-09-30 \mathrm{~T} 06: 34: 39$ \\
241 & $\mathrm{CP}$ & 91 & 177.3 & $2004-10-03 \mathrm{~T} 06: 23: 00$ \\
242 & $\mathrm{CP}$ & 2 & 89.3 & $2004-10-06 \mathrm{~T} 06: 10: 07$ \\
243 & $\mathrm{CP}$ & 4 & 107.0 & $2004-10-09 \mathrm{~T} 05: 56: 48$ \\
244 & $\mathrm{CP}$ & 1 & 69.0 & $2004-10-12 \mathrm{~T} 05: 43: 59$ \\
245 & $\mathrm{R}$ & 9 & 157.3 & $2004-10-15 \mathrm{~T} 05: 31: 01$ \\
246 & $\mathrm{R}$ & 14 & 108.0 & $2004-10-18 \mathrm{~T} 05: 16: 53$ \\
249 & $\mathrm{CP}$ & 2 & 111.1 & $2004-10-27 \mathrm{~T} 04: 42: 52$ \\
\hline $225-249$ & & 173 & 2177 & \\
\hline & & & & \\
\hline
\end{tabular}

${ }^{1}$ Excluding the huge outburst of October 5 (see text).

model (see e.g. Duncan \& Thompson 1992; Paczynski 1992; Thompson \& Duncan 1995), which involves slowly rotating $(P \sim 5-8 \mathrm{~s})$ highly magnetised $\left(B \sim 10^{15} \mathrm{G}\right)$ isolated neutron stars. The magnetar model is based on the fact that the rotational energy loss inferred from the spindown (in the $10^{-10}-10^{-13} \mathrm{~s} \mathrm{~s}^{-1}$ range) is not sufficient to power the persistent $\mathrm{X}$-ray luminosity. Hence it is the decay of the magnetic field itself that provides the necessary energy. In this framework the magnetic dissipation causes the neutron star crust to fracture. These fractures generate sudden shifts in the magnetospheric footprints, which trigger the generation of Alfvén waves, which in turn accelerate electrons above the pair-production threshold, resulting quickly in an optically thick pair-photon plasma. The cooling of this plasma generates the typical short bursts of soft $\gamma$-ray radiation. An alternative explanation for short bursts, proposed by Lyutikov (2003), does not involve crustal

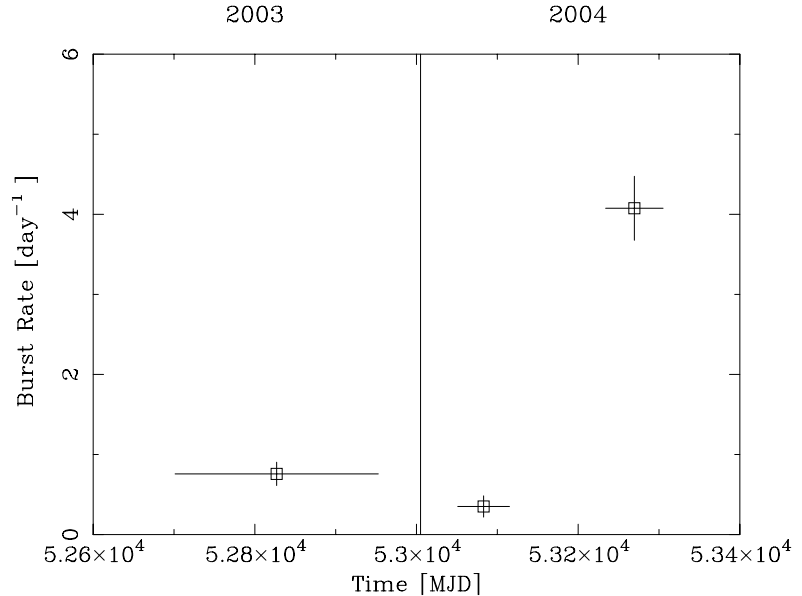

Fig. 1. SGR 1806-20 burst rate vs. time. Only the bursts with fluence $\geq 3 \times 10^{-8} \mathrm{erg} \mathrm{cm}^{-2}$ are considered. The three time periods correspond roughly to Fall 2003, Spring 2004 and Fall 2004.

fractures but simply local magnetic reconnection. The longer, much more energetic and rare flares are powered by a sudden reconfiguration of the magnetic field through magnetic reconnection (as in solar flares), and involve the entire neutron star magnetosphere.

After a period of quiescence, SGR 1806-20 became active in the summer of 2003 (Hurley et al. 2003). Its activity then increased in 2004 (see e.g. Mereghetti et al. 2004a; Golenetskii et al. 2004). A strong outburst (Mereghetti et al. 2004b) during which about one hundred short bursts were emitted in a few minutes occurred on October 5, 2004 (see Sect. 3.2). Finally a giant flare, whose energy ( $\left.\sim 10^{46} \mathrm{erg}\right)$ was two orders of magnitude larger than those of the previously recorded flares from SGR 0526-66 and SGR 1900+14, was emitted on December 27th 2004 (see e.g. Palmer et al. 2005; Mereghetti et al. 2005b; Hurley et al. 2005).

Here we report on all the short bursts detected by INTEGRAL in 2003 and 2004.

\section{Observations and data analysis}

The observations of this source obtained with the INTEGRAL satellite in September and October 2003, during a period of moderate bursting activity, have been reported earlier (see Götz et al. 2004a,b). We have re-analysed those data and, in addition, we report here on a much larger burst dataset. Excluding the bursts of the October 5th event (see below), we have detected and analysed 224 bursts. The observations are summarised in Table 1.

Our dataset represents the largest sample of short bursts imaged from SGR 1806-20 to date. As can be seen from Fig. 1, the burst activity of SGR 1806-20 increased significantly with time, and the source was particularly active during Fall 2004.

Here we concentrate on the data of the INTEGRAL imager IBIS (Ubertini et al. 2003). We do not use the data of the X-ray monitor JEM-X (Lund et al. 2003), since only a small fraction of the bursts were inside its smaller field of view and, owing to their brightness, they were affected by saturation and dead time effects. All the bursts were in the field of view of the SPI 
instrument (Vedrenne et al. 2003), but at energies below 300 $\mathrm{keV}$ SPI has a much smaller effective area compared to IBIS, which is therefore the most suitable instrument for detailed studies of short bursts.

IBIS is a coded mask telescope with a large field of view $\left(29^{\circ} \times 29^{\circ}\right.$ zero sensitivity, $9^{\circ} \times 9^{\circ}$ full sensitivity $)$. We used the low energy ( $15 \mathrm{keV}-1 \mathrm{MeV})$ detector layer ISGRI (Lebrun et al. 2003), composed of $128 \times 128$ independent CdTe detectors (pixels) yielding a geometric area of $\sim 1300 \mathrm{~cm}^{2}$ on axis. ISGRI operates in a photon-by-photon mode: this means that for each photon the energy, arrival time and interaction pixel are known. To identify the bursts we used the trigger information provided by the INTEGRAL Burst Alert System (IBAS; Mereghetti et al. 2003) for the Target of Opportunity (ToO), public and Core Program data. For the Galactic Centre Field data, we searched for the bursts by computing light curves with $10 \mathrm{~ms}$ time resolution and looking for significant excesses corresponding to the direction of SGR 1806-20 (for details see Molkov et al. 2005). All the bursts are unambiguously associated, within the typical $\sim 2$ ' location uncertainties, with the position of the X-ray counterpart of SGR 1806-20 (Kaplan et al. 2002).

For each burst we derived the $15-100 \mathrm{keV}$ light curve with $10 \mathrm{~ms}$ time resolution. This energy band was chosen because no emission above $100 \mathrm{keV}$ is detected in the typical short bursts. In order to increase the signal-to-noise ratio, the light curves have been extracted by selecting only the pixels illuminated by the source over at least half their surface. The background was estimated by fitting a constant to $2 \mathrm{~s}$ time intervals before and after the burst. The light curves were then corrected for vignetting, caused by the fact that the bursts were detected at different off-axis angles, and for ISGRI dead time. The detector is made of 8 identical modules and the good events dead time, $\tau$, for each module is $\sim 114 \mu \mathrm{s}$. For each burst, based on its position in the IBIS field of view, we determined the number of modules involved in the detection for at least half their surface, $M$, and applied the following dead time correction

$N_{\text {inc }}=\left(\frac{1}{N_{\text {rec }}}-\frac{\tau}{M}\right)^{-1}$,

where $N_{\text {rec }}$ is the recorded flux (cts/s) in each time bin and $N_{\text {inc }}$ is the reconstructed incident flux. By evaluating this relation, one sees that only for the bins with a ratio between the recorded flux and the maximum recordable flux $(M / \tau)$ larger than $0.3 \mathrm{a}$ significant correction is required. For the rest of the bins the dead time effect is less than a few percent. It turns out that only $5.3 \%$ of the bursts have peak fluxes above this limit and their light curves have been corrected accordingly.

These light curves have been used to determine the $T_{90} \mathrm{du}-$ rations (i.e. the time during which $90 \%$ of the burst fluence is accumulated), the peak fluxes (counts/bin) and the fluences (counts) of each burst. To convert the peak fluxes and fluences to physical units we have used the conversion factor 1 count $\mathrm{s}^{-1}=1.5 \times 10^{-10} \mathrm{erg} \mathrm{cm}^{-2} \mathrm{~s}^{-1}$, derived by the spectral analysis of the brightest bursts (see Götz et al. 2004a). These bursts are well fit with a Thermal Bremsstrahlung (TB) model with temperatures $(k T)$ between 30 and $40 \mathrm{keV}$. The above conversion factor is valid for the average temperature of $32 \mathrm{keV}$.

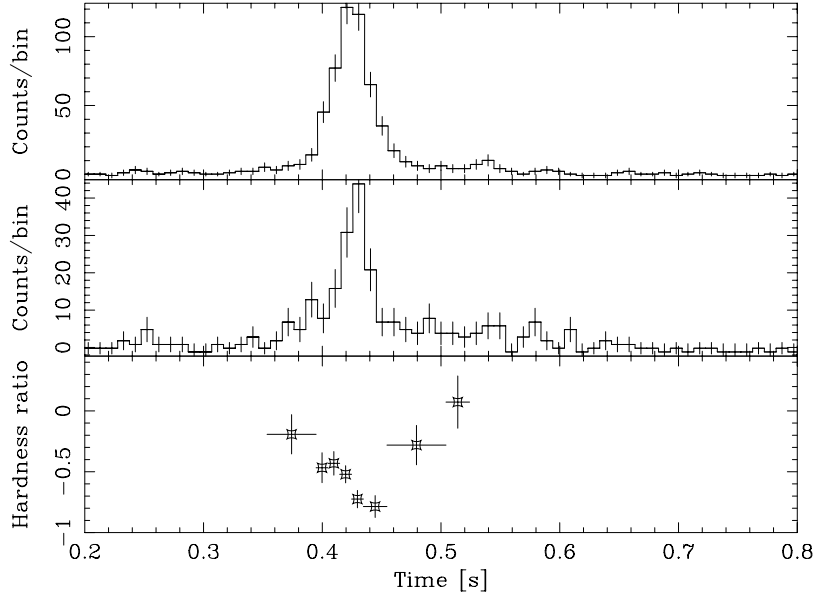

Fig. 2. IBIS/ISGRI light curves in the soft (20-40 keV, upper panel) and hard (40-100 keV, middle panel) energy range and hardness ratio (lower panel) for a short burst from SGR 1806-20. Time $=0$ corresponds to 2004-08-18T21:52:49.39 UT.

A variation of the temperature between the two extremes implies a variation of only $7 \%$ of the conversion factor. So we have applied it also to fainter bursts, under the assumption that the spectral shape is the same for all the bursts. We point out, however, that extracting the average spectrum of the 2003 bursts and comparing it to the average spectrum of a representative subsample of the bursts of Fall 2004, during which the source was more active, we find that the latter is marginally harder, but still within the above mentioned limits. Both average spectra are well represented by the TB model.

\section{Results}

\subsection{Spectral evolution}

Evidence for spectral evolution within the bursts of SGR 1806-20 was reported for the first time by Götz et al. (2004a), who found that some bursts show significant spectral evolution, while others, particularly those with a "flat topped" profile, do not. In addition, a hardness-intensity anticorrelation within the bursts of SGR 1806-20 was reported by the same authors.

We investigated the spectral evolution of the brightest bursts by computing the background subtracted light curves in two different energy bands (20-40 $(S)$ and 40-100 $(H) \mathrm{keV})$ and evaluating the hardness ratio, $H R=(H-S) /(H+S)$, in time bins with the same number of total counts. The energy bands used here are slightly different from those used in Götz et al. (2004a) because we realized that the individual pixels behave differently at energies between 15 and $20 \mathrm{keV}$. In our analysis the spectral evolution is confirmed: excluding "flat topped bursts", peaks tend to be softer than tails. Two good examples are shown in Figs. 2 and 3. However, a number of bursts show a less certain spectral evolution. By considering all the individual bins of all the bursts we derived the hardness $(H R)$ intensity $(I)$ distribution shown in Fig. 4. To investigate if the data are correlated, we computed the Spearman rank-order correlation coefficient of the 217 data 


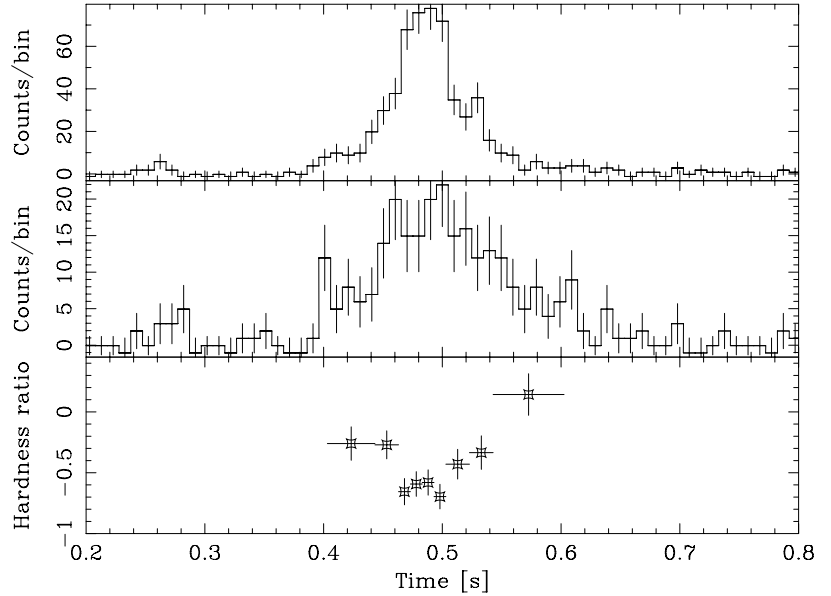

Fig. 3. IBIS/ISGRI light curves in the soft (20-40 keV, upper panel) and hard (40-100 keV, middle panel) energy range and hardness ratio (lower panel) for a short burst from SGR 1806-20. Time $=0$ corresponds to 2004-08-21T03:38:26.31 UT.

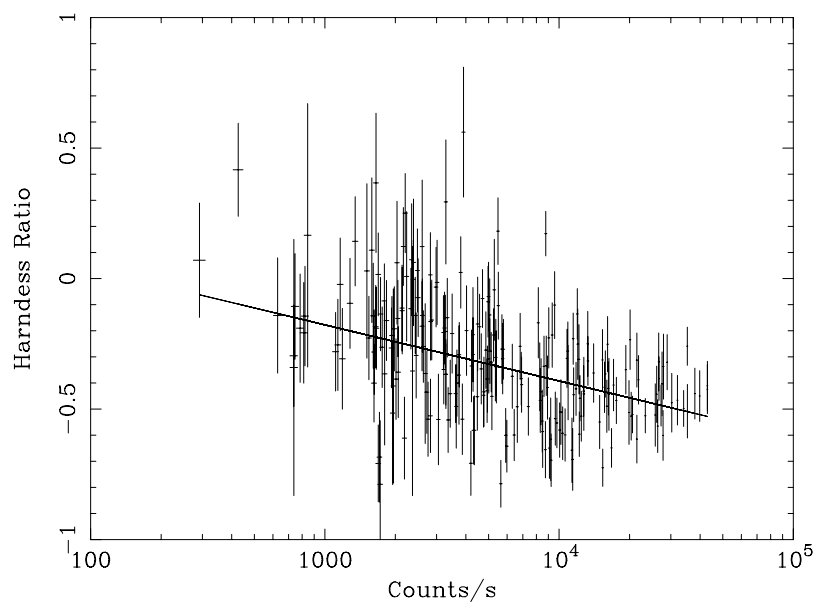

Fig. 4. Hardness ratio $((H-S) /(H+S))$ versus total count rate (20-100 keV, corrected for vignetting). The points are derived from the time resolved hardness ratios of the bursts with the best statistics. The line indicates the best fit with a linear function given in the text.

points, $R_{\mathrm{s}}$, which is -0.49 . This corresponds to a chance probability of $4 \times 10^{-15}(7.4 \sigma)$ that our distribution is due to uncorrelated data. According to an F-test the data are significantly $(8 \sigma)$ better described by a linear fit $(H R=0.47-0.22 \times \log (I))$ than by a constant. The parameters of the fit are very similar to those found by Götz et al. (2004a). So we can conclude that an anticorrelation between hardness and intensity is confirmed by our data. The inclusion or exclusion of flat-topped events from our analysis does not affect our results, since they represent a small fraction of the bursts.

\subsection{The large outburst of October 52004}

On October 5, 2004 IBAS triggered at 13:56:49 UT on the first of a series of bursts emitted from SGR 1806-20. The source remained active, emitting several tens of bursts, until 14:08:03 UT.
Figure 5 (lower panel) displays the initial and brightest part of the $\sim 11$ min long outburst. The time scale starts at 13:55:19 UT. We will refer to this time scale throughout our analysis. Two bright clusters of bursts are visible at $t \sim 100 \mathrm{~s}$ and $t \sim 280 \mathrm{~s}$. They are so bright that the satellite telemetry was partially saturated ( 0 counts in the plot) for about 10 and $20 \mathrm{~s}$ respectively and only part of the registered events could be sent to the ground.

The two bright clusters are shown in more detail in Figs. 7 and 8. Despite the presence of the telemetry gaps in the data, one can see that they consist of many short bursts, with significant variability down to $\sim 10 \mathrm{~ms}$ time scales. At least 75 short bursts, typical in terms of duration and shape, have been detected in IBIS/ISGRI data.

We have extracted a spectrum over the entire duration of the outburst (676 s integration time, see Fig. 6). A fit in the 20-300 keV band with a thermal bremsstrahlung model using XSPEC v11.2 (Arnaud et al. 1996) gives a good result $\left(\chi^{2}=\right.$ 39.64 for 40 d.o.f., allowing for 5\% systematics) and yields a temperature of $58 \pm 2 \mathrm{keV}$, higher than that usually measured for short bursts. A slightly worse but still acceptable fit ( $\chi^{2}=45.99$ for 40 d.o.f.) can be obtained using the sum of two black bodies, as proposed by Feroci et al. (2004) for the cumulative spectrum of short bursts from SGR 1900+14 and by Olive et al. (2004) for an intermediate duration burst from the same source. The temperatures of the two black bodies are $k T_{1}=5.8 \pm 0.4 \mathrm{keV}$ and $k T_{2}=18 \pm 0.9 \mathrm{keV}$. Both values are higher than those derived by Feroci et al. (2004) $\left(k T_{1}=\right.$ $3.3 \pm 0.1 \mathrm{keV}, k T_{2}=9.5 \pm 0.4 \mathrm{keV}$ ) and by Olive et al. (2004) $\left(k T_{1}=4.3 \pm 0.1 \mathrm{keV}, k T_{2}=9.9 \pm 0.3 \mathrm{keV}\right)$. On the other hand by fixing the temperatures to the values derived by these authors we get unacceptable fits. This is possibly due to the fact that their spectra extend to lower energy. In the ISGRI energy range this model does not improve the reduced $\chi^{2}$ of the fit and hence the results of the two black body fit have to be taken with care.

We computed the hardness ratios over the time periods of the two clusters of bright bursts $(t=88-108 \mathrm{~s}$ and $t=$ 260-293 s respectively). They indicate a clear hardening for the second cluster with respect to the first one. Even though the ISGRI detector partially saturates over these time intervals, the spectra still have enough counts to perform spectral analysis (see Figs. 7 and 8). We have hence extracted two spectra over these two time periods. Applying TB models to the spectra, we see that the temperature increases significantly with time. In fact for the first cluster of bursts we derive a temperature $(k T)$ of $39 \pm 2 \mathrm{keV}$, while for the second cluster we get $55.5 \pm 1.5 \mathrm{keV}$. We point out however (see Table 2) that the TB model does not fit the spectrum of the first cluster well. We divided this period into smaller time intervals and found that it is only the initial part $(t=89-92 \mathrm{~s})$, that cannot be represented by a TB model. Its spectrum is in fact much better fitted $\left(\chi^{2} /\right.$ d.o.f. $\left.=36.6 / 31\right)$ by a power law with photon index $\Gamma=2.80 \pm 0.05$. The overall spectrum is hence a mixture of different states, which may also explain the failure of the two black body model.

We have extracted 5 more spectra for 2 bright bursts at $t=205.45$ and $t=237 \mathrm{~s}$ and for 3 short $(\sim 0.1 \mathrm{~s})$ bursts at $t=406.4,560.25$ and $761.8 \mathrm{~s}$ respectively. All the 


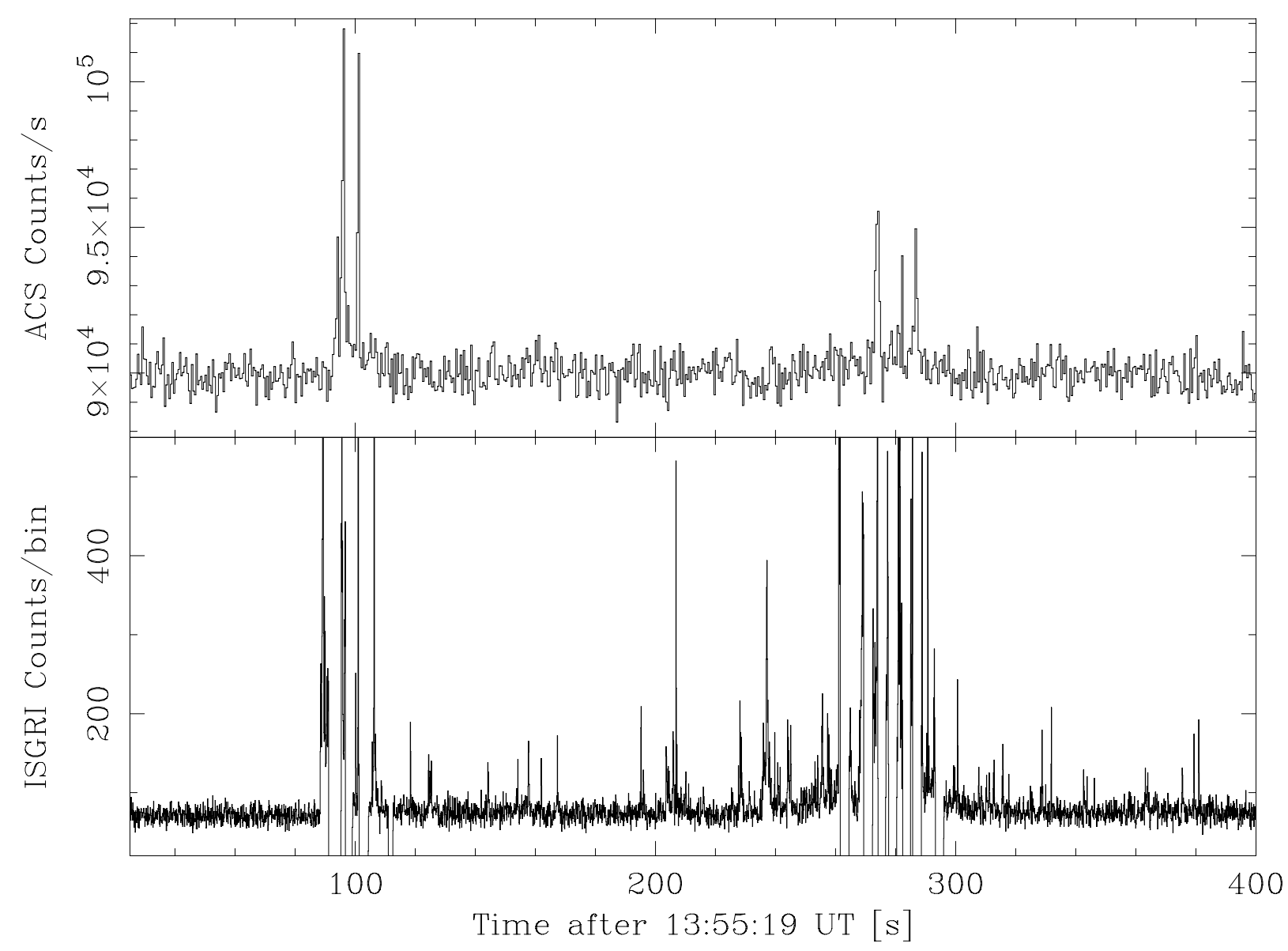

Fig. 5. Light curves of the initial part of the October 5, 2004 outburst of SGR 1806-20. Upper panel: light curve at energy greater than $\sim 80 \mathrm{keV}$ obtained with the SPI Anti-Coincidence System in bins of $0.5 \mathrm{~s}$. Bottom panel: light curve in the $15-200 \mathrm{keV}$ energy range obtained with the IBIS/ISGRI instrument (bin size $0.1 \mathrm{~s}$ ). The gaps in the IBIS/ISGRI light curve are due to saturation of the satellite telemetry.

Table 2. Temperatures, fluxes and statistical goodness of the fits of the different bursts obtained with the TB model.

\begin{tabular}{ccccc}
\hline \hline Time [s] & $\Delta t[\mathrm{~s}]$ & $k T[\mathrm{keV}]$ & $\begin{array}{c}\text { Flux 20-200 keV } \\
{\left[\mathrm{erg} \mathrm{cm}^{-2} \mathrm{~s}^{-1}\right]}\end{array}$ & $\chi^{2} /$ d.o.f. \\
\hline 88 & 20 & $39 \pm 2$ & $>^{1} 1.5 \times 10^{-7}$ & $118 / 36$ \\
205.45 & 4.1 & $61 \pm 8$ & $9.0 \times 10^{-8}$ & $33 / 28$ \\
237 & 4 & $74 \pm 7$ & $1.5 \times 10^{-7}$ & $39 / 33$ \\
260 & 33 & $55.5 \pm 1.5$ & $>^{1} 1.5 \times 10^{-7}$ & $52 / 40$ \\
406.4 & 0.2 & $34.5 \pm 11$ & $1.5 \times 10^{-7}$ & $6.8 / 6$ \\
560.25 & 0.45 & $65 \pm 7$ & $2.6 \times 10^{-7}$ & $23 / 20$ \\
761.8 & 0.2 & $44 \pm 7$ & $2.8 \times 10^{-7}$ & $19 / 16$ \\
\hline
\end{tabular}

1 The fluxes of the two bright clusters are not corrected for telemetry saturation.

spectra are better represented by TB models than by power laws. The derived temperatures are reported in Table 2. Although no specific trend can be identified, there are significant variations between the bursts' temperatures. The spectra of the bursts at $t=205.45,237$ and $560.25 \mathrm{~s}$ have very high temperatures, above $40 \mathrm{keV}$, which is the maximum temperature usually measured for short bursts. These events are similar in spectral hardness to two bursts detected from SGR 1900+14 following its giant flare (Woods et al. 1999).
The fluence of the entire outburst as measured by ISGRI is $1.5 \times 10^{-5} \mathrm{erg} \mathrm{cm}^{-2}$. This value is however heavily affected by the saturation of the brightest bursts and represents only a lower limit to the real fluence. In order to derive the overall fluence, we used the data provided by the Anti-Coincidence System (ACS) of the INTEGRAL spectrometer (SPI). As can be seen in Fig. 5 (upper panel), only the brightest bursts are visible in these data and hence they represent complementary information to the ISGRI data.

ACS data consist of the total count rate $(50 \mathrm{~ms}$ time resolution) above $80 \mathrm{keV}$ measured by the 91 bismuth germanate (BGO) scintillator crystals that surround the INTEGRAL spectrometer. The crystals are used as the anti-coincidence system of the spectrometer but are also capable of detecting high-energy transient events such as bright GRB and SGR bursts. We used the Monte Carlo package MGGPOD (Weidenspointner et al. 2005) and a detailed mass modelling of SPI and the whole satellite (Weidenspointner et al. 2003 and references therein) to derive the effective area of the ACS for the direction of SGR 1806-20. We computed the ACS light curve with a binsize of $0.5 \mathrm{~s}$ and estimated the background by fitting a constant value to all the data of the same pointing excluding the bursts. We used the background subtracted light curve to compute the fluence of each burst cluster in 


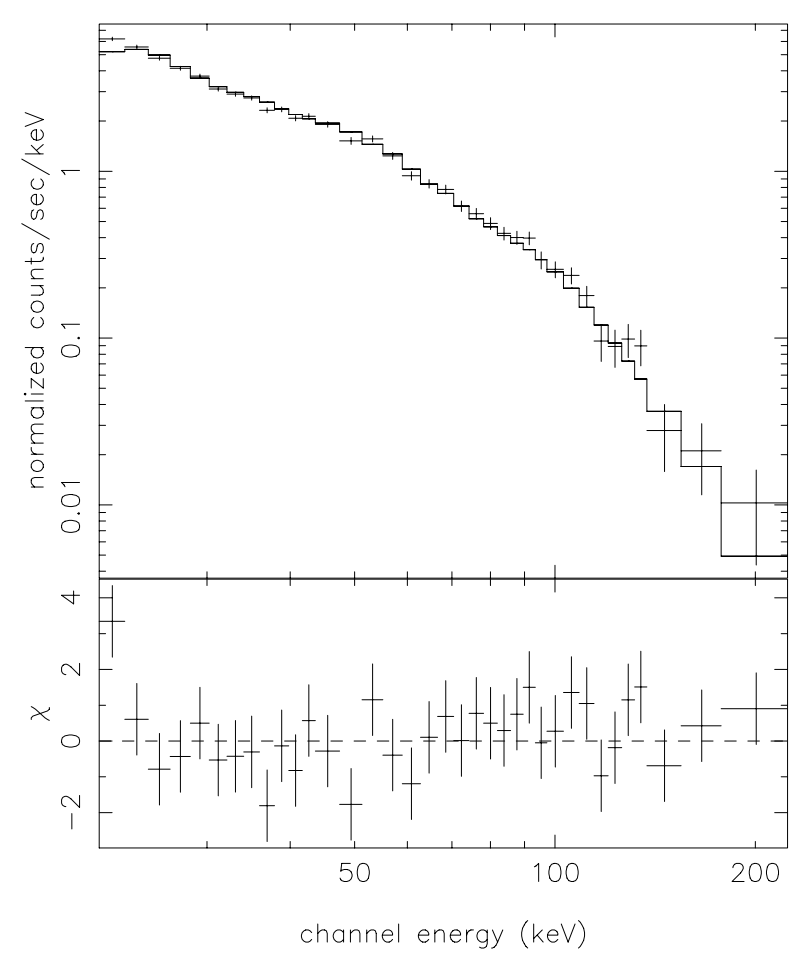

Fig. 6. Spectrum of the October 5 outburst of SGR 1806-20 measured with the IBIS/ISGRI instrument. Upper panel: data points and best fit with a thermal bremsstrahlung model with $k T=58 \mathrm{keV}$. Lower panel: residuals from the best fit in units of sigma.

counts. The ACS data do not provide any spectral information, so we computed the conversion factor to physical units based on the spectral shapes derived from ISGRI data and on the effective area computed through our simulations. The resulting fluences above $80 \mathrm{keV}$ are $1.2 \times 10^{-5}$ and $9.4 \times 10^{-6} \mathrm{erg}$ $\mathrm{cm}^{-2}$ for the first and second clusters respectively. Converting these fluences to the $15-100 \mathrm{keV}$ band one obtains $7.4 \times 10^{-5}$ and $3.2 \times 10^{-5} \mathrm{erg} \mathrm{cm}^{-2}$ respectively. By adding these results to the ones obtained for the ISGRI total spectrum, one can derive the total energy output during the whole event, which is $1.2 \times 10^{-4} \mathrm{erg} \mathrm{cm}^{-2}$. This corresponds to $3.25 \times 10^{42} \mathrm{erg}$ for an assumed distance of $15 \mathrm{kpc}$ (Corbel \& Eikenberry 2003).

\subsection{Number-intensity distribution}

The fluences of the 224 bursts derived above (excluding the ones detected during the October 5 th event) have been used to compute the number-intensity distribution $(\log N-\log S)$ of the bursts.

The experimental distribution deviates significantly from a single power-law (Fig. 9). This is first of all due to the fact that the source has been observed at different off-axis angles. The faintest bursts are missed when the source is observed at large off-axis angles. In order to correct for this effect we have computed the effective exposure of the source, taking into account the variation of sensitivity at various off-axis angles. This yields the exposure-corrected cumulative distribution shown by the dashed line in Fig. 9.
Since the numbers at each flux level are not statistically independent, one cannot use a simple $\chi^{2}$ minimisation approach to fit the cumulative number-intensity distribution. So we have used the unbinned detections and applied the Maximum Likelihood method (Crawford et al. 1970), assuming a single power-law distribution for the number-flux relation $(N(>S) \propto$ $S^{-\alpha}$ ). We have used only the part of the distribution where completeness was achieved (i.e. $S \geq 3 \times 10^{-8} \mathrm{erg} \mathrm{cm}^{-2}$ ). In this case the expression to be maximised is

$\mathcal{L}=T \ln \alpha-\alpha \sum_{i} \ln S_{i}-T \ln \left(1-b^{-\alpha}\right)$

where $S_{i}$ are the unbinned fluxes, $b$ is the ratio between the maximum and minimum values of the fluxes, and $T$ is the total number of bursts. This method yields $\alpha=0.91 \pm 0.09$. If a single power-law model is an adequate representation of data, the distribution of the quantities

$y_{i}=\frac{1-S_{i}^{-\alpha}}{1-b^{-\alpha}}$

should be uniform over the range $(0,1)$. In our case, a Kolmogorov-Smirnov (K-S) test shows that a power law is an appropriate model, yielding a probability of $98.8 \%$ that the data are well described by our model.

We then divided the bursts in two samples comprising 51 and 173 bursts respectively. The division is based on the periods of different activity. The first period ends at orbit 181 and the second one starts at orbit 225 (see Table 1). The two slopes derived with the Maximum Likelihood method are $\alpha=0.9 \pm 0.2$ for the low level activity period and $\alpha=0.88 \pm 0.11$ for the high level one. The two slopes are statistically consistent with each other and a K-S test shows that the probability that the two distributions are drawn from the same parent distribution is $93 \%$. Thus we conclude that the the relative fraction of bright and faint bursts is not influenced by the level of activity of the source.

\section{Discussion}

The bursts detected by INTEGRAL and presented here are common bursts as far as many aspects are concerned, such as durations ( $\sim 0.12 \mathrm{~s}$ on average), peak fluxes, fluences and spectra but are the faintest detected at these energies. Thanks to imaging we are confident that all of them were emitted by SGR 1806-20.

The good quality of our data has allowed us to study these bursts in detail. In particular, with respect to previous experiments, we have a better combination of sensitivity, timing and spectral capabilities also for faint events. Hence we have been able to confirm the early findings (see Götz et al. 2004a) on the spectral evolution of SGR 1806-20 short bursts. The fact that burst peaks tend to be softer than their tails leads to an overall hardness-intensity anti-correlation. This characteristic still has no clear explanation within the magnetar model.

The relatively large number of bursts has allowed us to constrain the shape and slope of the fluence distribution, which is well described by a single power-law on 


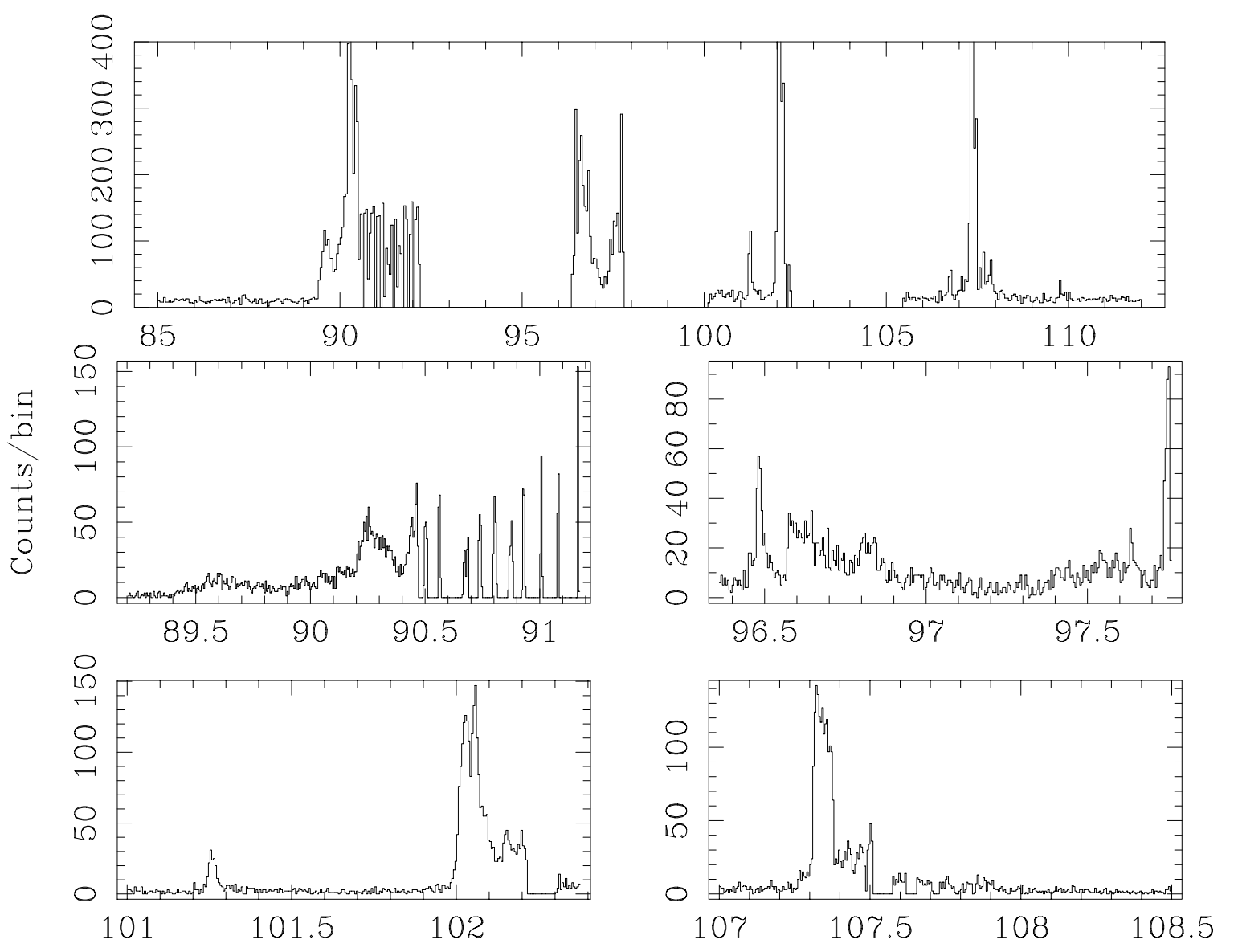

Fig. 7. IBIS/ISGRI light curves (15-200 keV) of the first cluster of bright bursts. Upper panel: overall light curve with $0.05 \mathrm{~s}$ resolution. The lower panels show some parts of the light curve in detail with a time resolution of $5 \mathrm{~ms}$. The time scale is in seconds and is the same as for Fig. 5.

over 2.5 orders of magnitudes. The INTEGRAL $\log N-$ $\log S$ has a slope of $0.91 \pm 0.09$, similar to that derived at larger fluences $\left(10^{-7}-10^{-5} \mathrm{erg} \mathrm{cm}^{-2}\right)$ with KONUS data (Aptekar et al. 2001). This slope is also compatible, within $2 \sigma$, with the $0.71 \pm 0.11$ obtained by combining BATSE and ICE data (Göğüş et al. 2000) over the $5 \times 10^{-8}-10^{-5} \mathrm{erg} \mathrm{cm}^{-2}$ fluence range. On the other hand our $\log N-\log S$ is significantly steeper than that obtained with $R X T E$, which has a slope of $0.43 \pm 0.06$ over the $10^{-10}-10^{-7} \mathrm{erg} \mathrm{\textrm {cm } ^ { - 2 }}$ fluence range (Göğüş et al. 2000). The difference between our slope and the $R X T E$ one, which extends to lower fluences, is significant: this may imply that there is a break in the distribution, but we point out that in the overlapping part of the two datasets $\left(6 \times 10^{-9}<S<10^{-7} \mathrm{erg} \mathrm{cm}^{-2}\right)$ the RXTE slope is clearly statistically rejected by our data. We can therefore conclude that, considering IBIS and BATSE/ICE data, a single powerlaw gives a good representation of the cumulative energy distribution of SGR 1806-20 bursts over 3.5 orders of magnitude.

Persistent emission in the $20-150 \mathrm{keV}$ range has been detected from SGR 1806-20 by integrating over long time intervals, part of the IBIS/ISGRI data reported here (Mereghetti et al. 2005a; Molkov et al. 2005). Although the persistent emission has a spectrum harder than that of typical bursts, it is worth to check whether a significant part of such persistent emission, could be due to the cumulative contribution of numerous bursts too faint to be detected individually. Integrating our $\log N-\log S$ distribution down to $S \sim 10^{-10} \mathrm{erg} \mathrm{cm}^{-2}$ we find that the burst contribution is at most a few percent $\left(\sim 10^{-12} \mathrm{erg} \mathrm{cm}^{-2} \mathrm{~s}^{-1}\right)$ of the total.

The hard and very energetic outburst of October 5th resembled a similar event seen in SGR $1900+14$ by KONUS on May 30, 1998 (Aptekar et al. 2001). In that case the outburst lasted $5 \mathrm{~min}$ and a total of 26 bursts were detected. The fluence was $3.5 \times 10^{-4} \mathrm{erg} \mathrm{cm}^{-2}$, similar to what we measure here, and the temperatures of the single bursts showed no particular correlation with time, but were on average at the high end of their distribution. In both sources these energetic outbursts, formed by a rapid sequence of relatively hard short bursts, preceded of a few months the occurrence of giant flares. The October 5th event fits in the trend of increasing source activity shown by SGR 1806-20 in the last two years and also manifested in the rise in luminosity and spectral hardness of the persistent emission at high (20-150 keV, Mereghetti et al. 2005a) and low (2-10 keV, Mereghetti et al. 2005c) energies. On the other hand, this peculiar event did not mark a peak or a turnover in the SGR activity. In fact the two XMM observations of SGR 1806-20 performed just before (September 6, 2004) and the day after this large outburst (as a ToO in response to it) yielded similar spectral parameters, fluxes and pulse profiles, and bursts were seen in both 


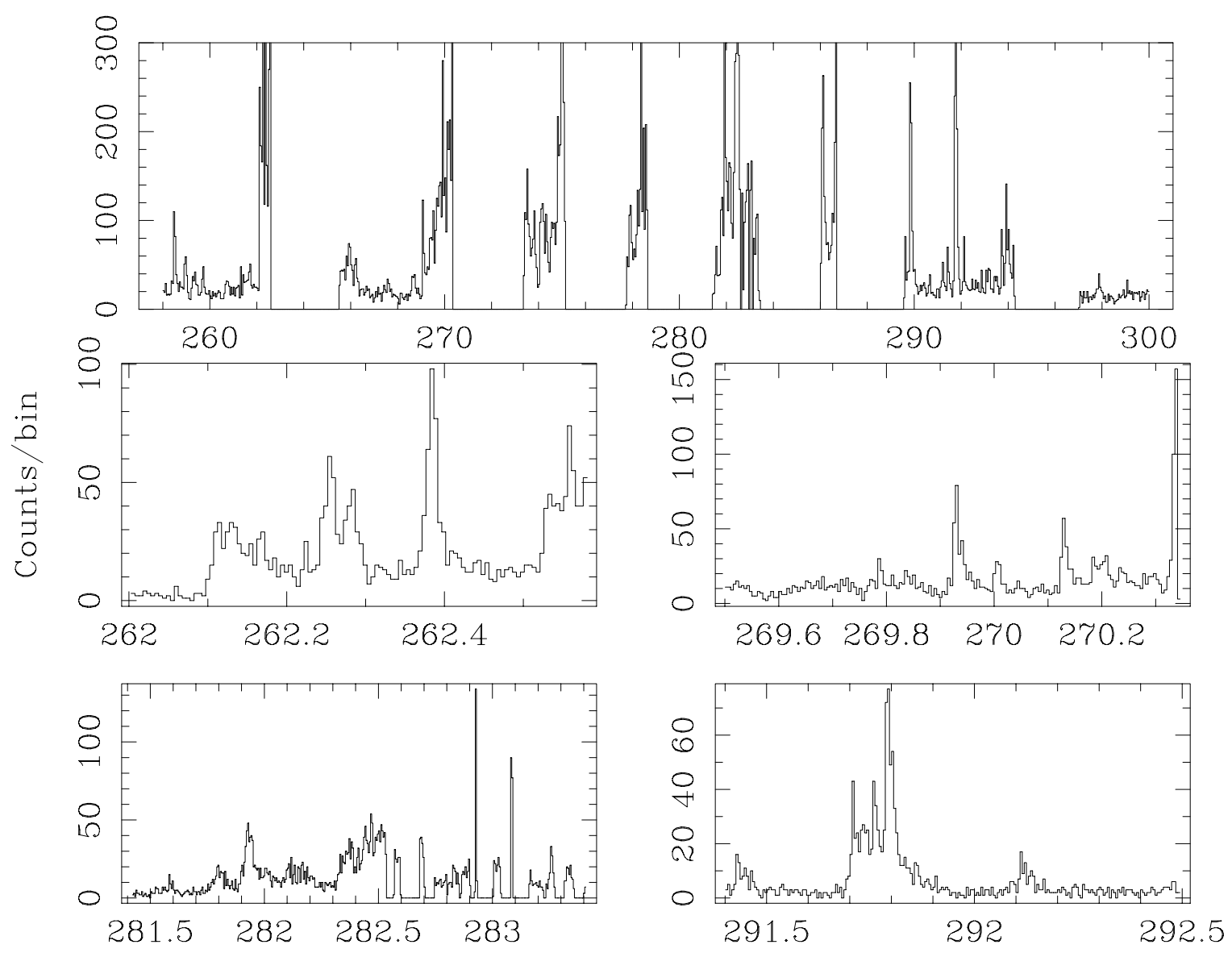

Fig. 8. IBIS/ISGRI light curves (15-200 keV) of the first cluster of bright bursts. Upper panel: overall light curve with $0.05 \mathrm{~s}$ resolution. The lower panels show some parts of the light curve in detail with a time resolution of $5 \mathrm{~ms}$. The time scale is in seconds and is the same as for Fig. 5.

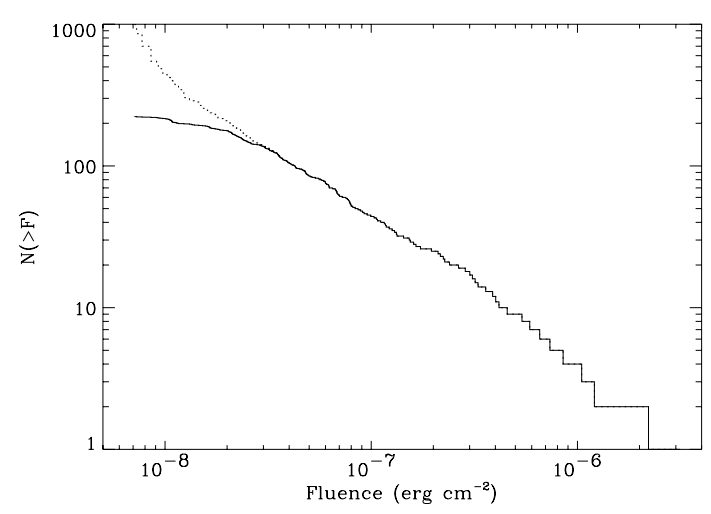

Fig. 9. Number-intensity distribution of all the bursts detected by INTEGRAL in 2003 and 2004. The continuous line represents the experimental data, while the dashed line represents the data corrected for the exposure.

observations (Mereghetti et al. 2005c). Also the INTEGRAL data indicate that the bursting activity remained high after this event. This also happened in 1998 to SGR $1900+14$, which after the May 30 event remained in a very active state leading to the August 27 giant flare (Aptekar et al. 2001).

These results can be explained in the framework of a recent evolution of the magnetar model: Lyutikov (2003) explains SGR bursts as generated by loss of magnetic equilibrium in the magnetosphere, in close analogy to solar flares: new current-carrying magnetic flux tubes rise continuously into the magnetosphere, driven by the deformations of the neutron star crust. This in turn generates an increasingly complicated magnetic field structure, which at some point becomes unstable to resistive reconnection. During these reconnection events, some of the magnetic energy carried by the currents associated with the magnetic flux tubes is dissipated. The large event described here can be explained by the simultaneous presence of different active regions (where the flux emergence is especially active) in the magnetosphere of the neutron star. In fact, a long outburst with multiple components is explained as the result of numerous avalanche-type reconnection events, as reconnection at one point may trigger reconnection at other points. This explains the fact that the outburst seems to be composed by the sum of several short bursts. This kind of event might indicate a particularly complicated phase of the magnetic field structure which eventually led to a global restructuring of the whole magnetosphere with the emission of the giant flare on December 27. This mode also suggests that short events are due to reconnection, while longer events have in addition a large contribution from the surface, heated by the precipitating particles, and are harder. This may explain the generally harder spectra observed.

Thus events like these release a small (compared to giant flares) fraction of the energy stored in the twisted magnetic 
field of the neutron star, not allowing the magnetic field to decay significantly. They are rather related to phases of high activity due to large crustal deformations (indicating that a large quantity of energy is still stored in magnetic form) and can be looked at as precursors of a major reconfiguration of the magnetic field.

Finally we would like to point out that after the giant flare of December 27, 2004, SGR 1806-20 has remained active, with a burst rate per day of $\sim 1.4$ between February 16 and April 28 2005 . This preliminary value indicates that the level of activity of the source is intermediate between 2003 and 2004. The emission of the giant flare, triggered by the reconfiguration of the magnetic field, has lowered the crustal stresses due to the magnetic dissipation (Thompson et al. 2002). A similar behaviour is seen in the X-ray band, where the persistent flux is intermediate between the 2003 and the 2004 observations (Mereghetti et al. 2005c; Rea et al. 2005; Tiengo et al. 2005).

\section{Conclusions}

We have presented the results of two years of INTEGRAL monitoring of SGR 1806-20. During this time period the source went from a $\gamma$-ray quiescent state into a very active one that culminated in the giant flare of December 27, 2004.

Our good quality data for low fluence bursts allowed us to establish that the number-density relation of the bursts is well represented by a single power law with index $\alpha=0.91 \pm 0.09$. Despite the increase in the rate of emitted bursts, the burst properties do not change significantly with time, neither does the slope of the number-density distribution. The spectral evolution of the bursts discovered in the 2003 data is confirmed also by this improved analysis of a larger sample of 224 bursts. The fact that bursts peaks tend to be softer than tails results in a hardness-intensity anti-correlation within the bursts.

On October 5, 2004, close to the peak of its activity SGR 1806-20 emitted within 10 min more than a hundred short bursts that had a spectrum slightly harder than usual and involved a total energy release of $3 \times 10^{42} \mathrm{erg}$. These high temperature bursts can be explained in the framework of the magnetar model as an avalanche-type reconnection event in the neutron star magnetosphere caused by a particularly complicated structure of the magnetic field. Events of this kind might be precursors of the major reconfiguration of the whole magnetic field of the neutron star causing large flares in SGRs.

Acknowledgements. We acknowledge the Italian Space Agency financial and programmatic support via contract $I / R / 046 / 04$. SeM thanks RFBR for grant $05-02-17465$. KH is grateful for support under the INTEGRAL US Guest Investigator program, NAG $5-13738$. The SPI-ACS is supported by the German "Ministerium für Bildung and Forschung" through the DLR grant 50.OG.9503.0.

\section{References}

Aptekar, R. L., Frederiks, D. D., Golenetskii, S. V., et al. 2001, ApJS, 137,227

Arnaud, K. A. 1996, Astronomical Data Analysis Software and Systems V, ASP Conf. Ser. 101, 17
Borkowski, J., Götz, D., Mereghetti, S., et al. 2004, GCN, 2920

Corbel, S., \& Eikenberry, S. S. 2003, A\&A, 419, 191

Crawford, D. F., Jauncey, D. L., \& Murdoch, H. S. 1970, ApJ, 162, 405

Duncan, R. C., \& Thompson, C. 1992, ApJ, 392, L9

Feroci, M., et al. 2004, ApJ, 612, 480

Göğüş, E., Woods, P. M., Kouveliotou, C., et al. 2000, ApJ, 532, L121

Golenetskii, S. V., Aptekar, R. L., Gur'yan, et al. 1987, Sov. Astron. Lett., 13, 166

Golenetskii, S., Aptekar, R., Mazets, E., et al. 2004, GCN, 2665

Götz, D., Mereghetti, S., Mirabel, F. I., \& Hurley, K. 2004a, A\&A, 417, L45

Götz, D., Mereghetti, S., Mirabel, F.I., et al. 2004b, in Proc. 5th INTEGRAL Workshop, ESA-SP 552, 615, [arXiv: astro-ph/0405135]

Hurley, K., Cline, T., Mazets, E., et al. 1999, Nature, 397, 41

Hurley, K. 2000, in AIP Conf. Proc. 526, 5th Hunstville Symp. Gamma-Ray Bursts, ed. R. M. Kippen, R. S. Mallozzi, \& G. F. Fishman (New York: AIP), 763

Hurley, K., Atteia, J.-L., Kawai, N., et al. 2003, GCN, 2308

Hurley, K., Boggs, S. E., \& Smith, D. M. 2005, Nature, 434, 1098

Kaplan, D. L., Fox, D. W., Kulkarni, S. R., et al. 2002, ApJ, 564, 935

Lebrun, F., Leray, J. P., Lavocat, P., et al. 2003, A\&A, 411, L141

Lund, N., Budtz-Jørgensen, C., Westergaard, et al. 2003, A\&A, 411, L231

Lyutikov, M. 2003, MNRAS, 346, 540

Mereghetti, S., Götz, D., Borkowski, J., Walter, R., \& Pedersen, H. 2003, A\&A, 411, L291

Mereghetti, S., Götz, D., Mowlavi, N., Shaw, S., \& Hurley, K. 2004a, GCN, 2647

Mereghetti, S., Götz, D., Borkowski, J., Beck, M., \& Mirabel, F. 2004b, GCN, 2763

Mereghetti, S., Götz, D., Mirabel, I. F., \& Hurley, K. 2005a, A\&A, 433, L9

Mereghetti, S., Götz, D., von Kienlin, A., et al. 2005b, ApJ, 624, L105

Mereghetti, S., Tiengo, A., Esposito, P., et al. 2005c, ApJ, 628, 938

Mazets, E. P., Golenetskii, S. V., Ilinskii, V. N., et al. 1979, Nature, 282,587

Molkov, S., Hurley, K., Sunyaev, R., et al. 2005, A\&A, 433, L13

Olive, J.-F., Hurley, K., Sakamoto, T., et al. 2004, ApJ, 616, 1148

Paczynski, B. 1992, AcA, 42, 145

D. M., Palmer, S., Barthelmy, N., Gehrels, et al. 2005, Nature, 434, 1107

Rea, N., Tiengo, A., Mereghetti, S., et al. 2005, ApJ, 627, L133

Thompson, C., \& Duncan, R.C. 1995, MNRAS, 275, 255

Thompson, C., Lyutikov, M., \& Kulkarni, S. R. 2002, ApJ, 574, 332

Tiengo, A., Esposito, P., Mereghetti, S., et al. 2005, A\&A, 440, L63

Ubertini, P., Lebrun, F., Di Cocco, G., et al. 2003, A\&A, 411, L131

Vedrenne, G., Roques, J.-P., Schönfelder, V., et al. 2003, A\&A, 411, L63

Weidenspointner, G., Kiener, J., Gros, M., et al. 2003, A\&A, 411, L113

Weidenspointner, G., et al. 2005, ApJS, 156, 69

Winkler, C, Courvoisier, T.J.-L., Di Cocco G., et al. 2003, A\&A, 411, L1

Woods, P. M., Kouveliotou, C., van Paradijs, J., et al. 1999, ApJ, 527, L47

Woods, P. M., \& Thompson, C. 2004, in Compact Stellar X-ray Sources, W. H. G. Lewin and M. van der Klis [arXiv: astro-ph/0406133] 